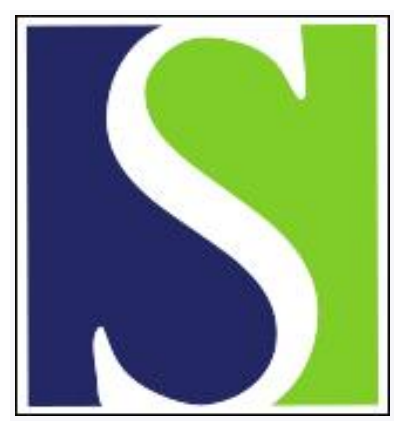

Scand J Work Environ Health 1985;11(3):223-227

https://doi.org/10.5271/sjweh.2231

Issue date: Jun 1985

\title{
Current problems of nonionizing radiation.
}

by Izmerov NF

This article in PubMed: www.ncbi.nlm.nih.gov/pubmed/4035325

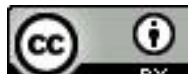

This work is licensed under a Creative Commons Attribution 4.0 International License 


\title{
Current problems of nonionizing radiation
}

\author{
by Nikolai F Izmerov, Corresponding Member of the USSR Academy of Medical Sciences
}

\begin{abstract}
IZMEROV NF. Current problems of nonionizing radiation. Scand J Work Environ Health 11 (1985) 223-227. Electromagnetic radiation in the radiofrequency range is the most common type of nonionizing radiation. The accelerated development in telecommunications and the introduction of power transmission lines, as well as glue drying and plastic heating in the wood and plastic industries, cause both occupational and nonoccupational systematic exposure to electromagnetic radiation in the radiofrequency range. For the hygienic rating of radiowave exposure it is necessary to use biological models to evaluate, in depth, the mechanisms and effects of electromagnetic radiation. The ultra- and superhigh frequency range is the most active in causing biological responses. The high frequency range is less active. Physical characteristics (continuous and impulse) and the combined effect of the electromagnetic field with other factors, eg, elevated air temperature, etc, have an effect on biological responses.
\end{abstract}

Key terms: biological effects, nonionizing radiation, physical characteristics, rating.

Several years ago it was generally supposed that environmental pollution was caused by chemicals and aerosols. Currently, however, the problem of the effects of physical factors on the general population and, particularly, on workers is among the most urgent of hygienic and ecological questions. Among the factors playing a major role in environmental pollution, physical factors (noise, vibration, ionizing and nonionizing radiation) have become more and more hazardous.

Five of the 31 environmental health criteria issued by the World Health Organization (WHO) are devoted to physical factors (noise, ultraviolet radiation, electromagnetic radiation in the radiofrequency range and microwaves, lasers, and optical radiation). Criteria on extremely low frequencies of electric and magnetic fields (up to $300 \mathrm{GHz}$ ) have also been prepared.

\section{Electromagnetic radiation - An occupational and environmental problem}

Without aiming at a comprehensive detailed description of the role of the different physical factors affecting man, it seems relevant to stress some aspects of the problem concerning nonionizing radiation. Different types of electromagnetic radiation are present in the occupational environment. Characteristically, in the course of rapid technical progress, the use of electromagnetic radiation has increased so that the problem which was earlier limited to occupa-

1 Corresponding Member of the USSR Academy of Medical Sciences, Institute of Industrial Hygiene and Occupational Diseases of the USSR AMS, Moscow, Union of Soviet Socialist Republics.

Reprint requests to: Prof NF Izmerov, Institute of Industrial Hygiene and Occupational Diseases of the USSR AMS, Prospect Budennogo 31, Moscow 105275, USSR. tional hygiene is now present also in the environment and with respect to ecology. Electromagnetic radiation in the radiofrequency range has been considered a potentially hazardous occupational factor and has been studied in detail during the past several decades.

At present a systematic increase in the use of electromagnetic energy in the radiofrequency range is being observed in all spheres of human activity. Electromagnetic radiation is used in many different operations, from the heating, welding and melting of metals and the processing of timber and plastic, to the creation of high-temperature plasma and the use of extremely high-frequency generators in modern electronic computers. The use of electromagnetic energy makes possible the production of principally new technological and manufacturing processes. The use and consumption of electronic devices and electromagnetic energy are rapidly developing, the result being a greater distribution of more powerful radiotechnical devices and electronic apparatus with new frequency ranges and generation patterns.

Simultaneously, the accelerated development of telecommunications and the introduction of power transmission lines cause systematic exposure of a significant portion of the world population to electromagnetic radiation in the radiofrequency range. One characteristic of this development is that the border between occupational and nonoccupational irradiation levels is becoming less pronounced.

\section{Physical characteristics of electromagnetic radiation}

Intense experimental study of the biological effect of electromagnetic fields in the radiofrequency range as an occupational factor started from the investigation of their thermal effects. It was proved that, when the wavelength decreases, the thermogenous effect in- 
creases. Later, many Soviet and other scientists convincingly showed that intensities of electromagnetic radiation that did not cause a thermal effect could result in other significant biological effects. Such biological effects influence an organism's functional activities unfavorably.

The effect of electromagnetic fields on an organism depends on such physical parameters as wavelength, radiation intensity, and irradiation pattern (continuous or impulse), as well as on duration of exposure and the combined effect of other occupational environmental factors (eg, elevated air temperature, X-ray radiation, etc). The extremely high frequency (EHF) range is the most biologically active; it is followed by the ultrahigh frequency (UHF) range. The high frequency (HF) range is less active. Thus the shorter the wavelength, the greater the biological effect.

The pattern of generation - continuous or impulse - plays a special role. Today, essentially, there is no unanimous opinion on the effects of continuous versus impulse irradiation on the organism. Some scientists are inclined to think that the interrupted pattern is more favorable. Others, however, believe that impulse irradiation may increase the effect of the electromagnetic field.

Data on the combined effects of electromagnetic radiation and other occupational factors are definitely of interest. These data enable scientists to approach the assessment of the real hazard of workers' occupational exposure to this factor. Thus, for example, it has been determined that joint exposure to electromagnetic radiation, an elevated air temperature, and soft $\mathrm{X}$-ray radiation causes a certain increase in effect. The study of the combined effect of EHF and soft X-ray radiation made it possible to determine the dependence of the character of the biological effect on the intensity of exposure. Thus, during low-intensity exposure to $\mathrm{X}$-ray and thermal radiation, EHF if the main factor in the combination.

The mechanisms of the biological effects of electromagnetic radiation should be thoroughly explored. The activities of the International Committee on Non-Ionizing Radiation (which is organized within the framework of the International Commission on Radiological Protection) are very demonstrative in this respect. The analysis of biological effects caused by exposure to different types of nonionizing radiation is one of the tasks of this committee. It should be stressed that, at present, the number of countries and specialists investigating this problem is increasing. In this connection the digest of works "Biological Effects and Dosimetry of Non-Ionizing Radiation" should be mentioned. Leading scientists from the United States, Canada, and Western Europe took part in its publication. In this material much attention is paid to theoretical problems - to the analysis of the interaction between the energy of electromagnetic radiation and substance.

\section{Biological effects of radiofrequency radiation}

An analysis of numerous investigations on the determination of various aspects of the biomechanisms of electromagnetic radiation makes possible the suggestion that electromagnetic fields directly affect tissues and organs. They also produce neurohumoral and reflectory effects. The three mechanisms are probably equal, and the contribution of each is determined by several physical and biological parameters.

Lately the effect of electromagnetic waves on some systems of an organism has become the center of scientists' attention. It has been determined that, for example, the depression of the function of the neocortex is the most characteristic consequence of chronic exposure to microwaves of nonthermogenous intensity. The changes in an electroencephalogram correspond to the depression of conditional (reflectory) activity of animals during the irradiation process.

The immune system is plastic. It reacts quickly to the weakest environmental changes, including electromagnetic fields of the nonionizing part of the spectrum. A study of changes in the immune system, determined as changes in phagocytic and bacteriocytic blood function, during exposure to all radiowave ranges of low intensity indicated that activation is substituted by depression.

The condition of reproductive function is one of the significant indicators of the biological effect of electromagnetic radiation in the radiofrequency range. Data on the high sensitivity of the reproductive system to radiowaves have been received.

Currently the genetic effect of electromagnetic radiation and its effects on growth and development, the hemopoietic system, and the cardiovascular system, as well as some other aspects of the biological effects of electromagnetic radiation, are also actively being studied.

Naturally knowledge of the biological mechanisms is necessary for the successful prevention of their unfavorable effects. It forms the basis for the effective struggle to combat them. That is particularly why the biological effects of low-intensity irradiation are under such intense research today. Such investigations make it possible to understand the pathogenic essence of functional changes determined in clinical examinations.

\section{Rating the harmfulness of radiofrequency radiation}

Maximum permissible levels of radiowave irradiation should be regarded as the main method to prevent the unfavorable effects of both electromagnetic radiation in the radiofrequency range and other potentially hazardous occupational factors.

In the Soviet Union the hygienic regulation of radiofrequency effects is based on the threshold of 
the harmful effect of a factor with a coefficient of hygienic accumulation as the criterion of harmfulness. The maximum permissible levels have been determined for the whole radiofrequency range on the basis of a complex made up of hygienic, clinical, and experimental studies. Moreover, as knowledge about various mechanisms deepens and the basis of the hygienic ratings becomes more accurate, the existing standards are revised. Besides, the introduction of new frequency ranges and new radiation regimes into industry creates a necessity for their hygienic regulation. The threshold of a harmful effect occurs when a combination of rated parameters causes changes in an organism, these changes are characterized by the presence of one or more of the following signs: (i) a qualitative reconstruction of living processes, (ii) quantitative changes in living processes exceeding the limits of physiological norms, and (iii) changes in living processes which are caused by cumulative effects of previous exposure and the degree of which will exceed permissible quantitative limits if exposure continues.

Knowledge of biological effects and the emissions of radiating devices, so-called radiobiology of nonionizing radiation, is the basis of modern hygienic ratings of radiowave exposure. Determining the quantitative connection between the emission and the absorption of electromagnetic energy and examining the distribution peculiarities of electromagnetic radiation in different frequencies inside a biological object with regard to the physical properties of the object's tissues in relation to its form, geometric dimensions, etc, is especially important. It is also significant to determine the energy converted to other types of energy in biological substratum and the peculiarities of biophysical processes in relation to the quantity of the absorbed energy. New information on the peculiarities of absorption and the distribution of energy in the biological system will greatly facilitate the scientific substantiation of the principles of extrapolating experimental data to man.

At present the evaluation and elaboration of hygienic norms for electromagnetic radiation is taking place in the field of occupational hygiene. These norms make it possible to rate electromagnetic radiation in relation to its exposure characteristics (frequency range, intensity, duration), generation pattern (continuous or impulse), and irradiation type (whole-body or local, permanent or intermittent). The possibility of the joint effect of electromagnetic radiation and other unfavorable environmental factors is taken into account in these standards.

One more aspect of the hygienic rating should also be considered. The results of clinical examinations are very important for elaborating and evaluating maximum permissible levels. Unfortunately, as yet, there is no complete understanding among scientists working in this field. There are certain differences both in the interpretation of the clinical changes caused by the exposure and the levels of irradiation permitted by the standards of different countries (see table 1). In my opinion it would be expedient to conduct epidemiologic studies in the form of special, unified programs. These studies should be aimed at establishing an objective classification of the clinical manifestations of radiation effects and at investigating the immediate and long-term effects of exposure. Special attention should be paid to the connection between such exposure and health changes.

It has already been stressed that sources of electromagnetic radiation can be dangerous not only for workers, but also for the general population because of the wide use of radioelectronic devices in various spheres of human activities. This use is characteristic for practically all economically developed countries. According to data from the Environmental Protection Agency in the United States, about $1 \%$ of the world population is exposed to electromagnetic radiation with an intensity of more than $1 \mu \mathrm{W} / \mathrm{cm}^{2}$. Such a situation makes joint elaboration of the problems of industrial and environmental hygiene a necessity. Microwave electromagnetic fields of nonthermal intensity are especially interesting for investigation. It should be stressed that the problem of microwave radiation in relation to both biologically and hygienically significant environmental factors is being worked out rather actively. In these studies attention has been paid primarily to the investigation of biological effects and the assessment of the significance of the reactions of the organism. Data on the relationship between the biological effect of microwave radiation and its manifestation, duration, and pattern of action are being used to establish maximum permissible levels of exposure for the general population. The genetic hazard of microwaves of nonthermal intensity is also being assessed. Naturally, the results of such studies are very interesting for occupational hygiene.

\section{Biological effects of infrared and ultraviolet radiation}

Infrared and ultraviolet radiation also belongs to the range of nonionizing radiation. It seems that the biological effect of these frequencies should already have been well investigated. However, the introduction of new technological processes changes significantly the conditions of exposure to such factors. It results in the appearance of new and different combinations, which modify the effects. For example, the use of low-temperature plasma $\left(15000-20000^{\circ} \mathrm{C}\right)$ is connected with intense ultraviolet radiation. As has been determined, ultraviolet radiation in the range with a wavelength of $1900-3000 \AA$ causes mutagenic and carcinogenic effects. During the application of low-temperature plasma, intense noise and ultrasound appear, together with intense ultraviolet radiation. Air ionization also takes place. The use of 
Table 1. Comparative characteristics of levels of microwave effects permitted in various countries. (MPL $=$ maximum permissible level, $M P L_{\text {lim }}$ = ceiling level, $P D=$ power density, ANSI = American National Standards Institute)

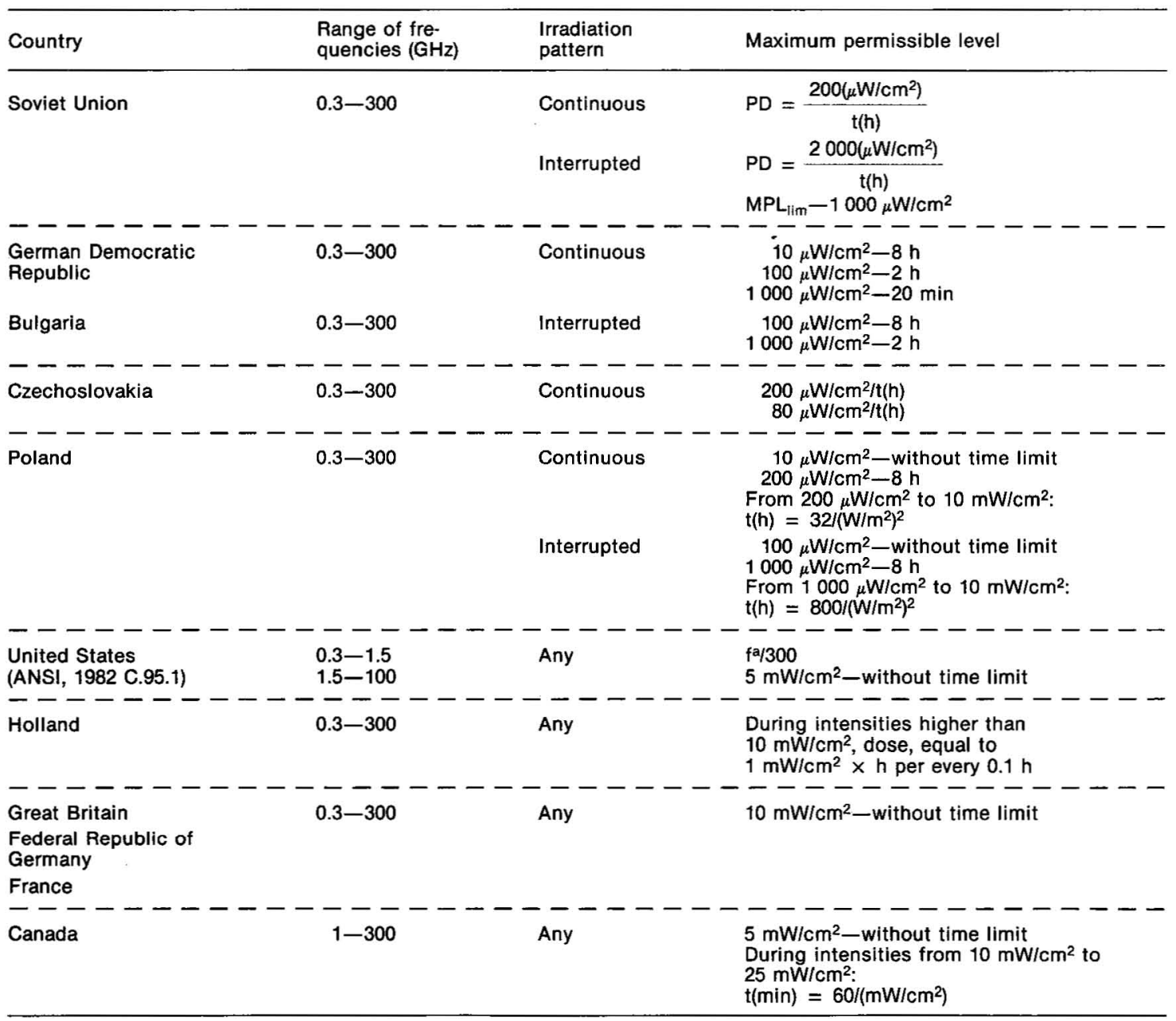

a $f=$ frequency in megahertz.

electromagnetic radiation in the radiofrequency range and soft $\mathrm{X}$-ray radiation are spreading. Therefore further study and the prevention of the unfavorable effects of ultraviolet radiation, particularly in combination with other occupational factors, are necessary. The problems of setting hygienic standards with regard to possible mutagenic and carcinogenic effects should also be considered.

\section{Biological effects of laser radiation}

Optic quantum generators and lasers form a completely new ecological problem. Other types of electromagnetic radiation of the whole specturm of frequencies have been affecting living organisms throughout evolution, but there is no analogue in nature for laser radiation. Lasers were created only a little more than 25 years ago, but their use and their rate of introduction into industry are so very high that, at present, it is difficult to enumerate those fields of science and technology in which lasers are not or will not be applied. Metal processing, defectoscopy, geodesy, construction, biology, medicine, control over chemical reactions, and thermonuclear synthesis form a partial list of areas utilizing a modern application of the laser ray. Naturally laser radiation has been thoroughly assessed from the point of view of possible unfavorable effects on workers. Numerous epidemiologic and experimental studies indicate that laser radiation is a biologically active physical factor and can be dangerous for man. What is important is that, during work with a source of laser radiation, a complex of factors can influence man. All the potentially hazardous factors can be conditionally divided into two groups. The first group comprises factors resulting from the work of the laser itself. The degree of manifestation of such factors depends on physical-technical parameters of the source (direct laser radiation, impulse light flashes, ultraviolet radiation, ozone and nitrogen 
oxide, noise, soft $\mathrm{X}$-ray radiation, electromagnetic radiation in the radiofrequency range, and toxic fluids). The second group is formed from factors resulting from the interaction between the laser ray and the processed materials or the various elements necessary for manipulating the laser ray (diffuse or mirror reflection of laser radiation, air environmental pollution with aerosols, electric fields of high intensity, impulse light flashes, etc).

\section{Rating the harmfulness of laser radiation}

The prevention of the harmful effects of laser radiation on workers is closely connected with the study of its biological effects. The eye is an organ that selectively reacts to laser radiation. Recently, data on the possibility of carcinogenic and mutagenic effects of laser radiation appeared in the literature. In particular, in the works of American scientists, data appear on the development of malignant tumors in scars after skin burns. It was also determined that functional changes of the central nervous system, the cardiovascular system, the endocrine system, etc, can develop in people working at laser installations, even during exposure to laser radiation of low intensities. Therefore, while taking hygienic measures, one must also remember that the possibility of harmful effects of laser energy is not all that should be accounted for. Scientists should also bear in mind that this factor is an inadequate irritator of the organism, even at low intensities. Besides, there are data on the increase of biological effects during combined exposure with other occupational environmental factors.

Thus far definite success has been achieved in proposing hygienic standards on laser radiation. Not only has the influence of the various wavelengths, the duration of exposure, various characteristics, and periodicity been taken into account in respect to the susceptibility of the visual organ, but also other biological effects (such as nonspecific changes appearing in an organism as a result of radiation) are considered in these hygienic standards. In addition maximum permissible levels have been determined for skin exposure. Thus the hygienic standards worked out on the basis of the comprehensive study of the character and mechanism of the biological effect of nonionizing radiation are the most important part of the system of measures protecting from its unfavorable effect. At the same time, the complex of protective measures providing safety for workers under occupational conditions is far broader.

\section{Conclusions}

The methods for approaching the hazardousness of nonionizing radiation should be considered more thoroughly. Not only should the procedures used to assess the hazard of a factor be improved, but it is also necessary to work out devices with which to perform measurements in a wide range of frequencies during various patterns of generation and exposure. Also important are effective engineering and technical decisions, hygienic assessment of the sources of nonionizing radiation during their design, and the medical surveillance of workers' health. These problems should be solved by occupational hygienic institutions or engineering and technical inspections organized to control work conditions.

In this report those aspects of the problem are mentioned which demand the attention of the world scientific community. Their solution will improve the occupational and general environment and prevent possible harmful effects on the health of people. 